OPEN ACCESS

Edited by:

Yufeng Dong,

Louisiana State University Health

Sciences Center, United States

Reviewed by:

Francesco Grassi,

Rizzoli Orthopedic Institute (IRCCS),

Italy

Rosemary Dziak,

University at Buffalo, United States

*Correspondence:

Xin Qi

qixin19871012@163.com

Dan Han

hdandqx@163.com

tThese authors have contributed equally to this work

Specialty section:

This article was submitted to Tissue Engineering and Regenerative Medicine,

a section of the journal Frontiers in Bioengineering and Biotechnology

Received: 25 May 2020 Accepted: 28 October 2020 Published: 19 November 2020

Citation:

Ying C, Wang R, Wang Z, Tao J, Yin W, Zhang J, Yi C, Qi X and Han D

(2020) BMSC-Exosomes Carry

Mutant HIF-1a for Improving Angiogenesis and Osteogenesis in

Critical-Sized Calvarial Defects. Front. Bioeng. Biotechnol. 8:565561. doi: 10.3389/fbioe.2020.565561

\section{BMSC-Exosomes Carry Mutant HIF-1 $\alpha$ for Improving Angiogenesis and Osteogenesis in Critical-Sized Calvarial Defects}

\author{
Chenting Ying ${ }^{1 \dagger}$, Rui Wang ${ }^{2 t}$, Zhenlin Wang ${ }^{3}$, Jie Tao', Wenjing Yin' ${ }^{4}$, Jieyuan Zhang ${ }^{4}$, \\ Chengqing Yi ${ }^{1}$, Xin Qi ${ }^{1 *}$ and Dan Han ${ }^{5 *}$ \\ ${ }^{1}$ Department of Orthopedics, Shanghai General Hospital, Shanghai Jiao Tong University School of Medicine, Shanghai, \\ China, ${ }^{2}$ Department of Critical Care Medicine, Shanghai General Hospital, Shanghai Jiao Tong University School \\ of Medicine, Shanghai, China, ${ }^{3}$ Department of Neurosurgery, Shanghai General Hospital, Shanghai Jiao Tong University \\ School of Medicine, Shanghai, China, ' ${ }^{4}$ Department of Orthopedics, Shanghai Jiao Tong University Affiliated Sixth People's \\ Hospital, Shanghai, China, ${ }^{5}$ Department of Emergency Medicine and Intensive Care, Shanghai Songjiang Clinical Medical \\ College of Naniing Medical University, Shanghai, China
}

Repair and reconstruction of critical-sized bone defects has always been a difficult task in orthopedics. Hypoxia inducible factor- $1 \alpha$ (HIF-1 $1 \alpha)$ plays an important role in bone defect repair, it has the dual function of promoting osteogenesis and vascular regeneration, but it is quickly degraded by the body under normoxic conditions. Previously we prepared mutant HIF-1 $1 \alpha$, which has been shown to efficiently maintain cellular expression under normoxic conditions. In this study, we evaluated for the first time the role of exosomes of rat bone marrow mesenchymal stem cell carry mutant HIF-1 $\alpha$ (BMSC-Exos-HIF1 $\alpha$ ) in repairing critical-sized bone defects. Evaluation of the effects of BMSC-Exos-HIF1 $\alpha$ on bone marrow mesenchymal stem cells (BMSCs) proliferation and osteogenic differentiation by cell proliferation assay, alkaline phosphatase activity assay, alizarin red staining, real-time quantitative polymerase chain reaction. BMSC-Exos-HIF1 $\alpha$ was loaded onto the $\beta$-TCP stent implanted in the bone defect area using a rat cranial critical-sized bone defect model, and new bone formation and neovascularization were detected in vivo by micro-CT, fluorescence labeling analysis, Microfil perfusion, histology and immunohistochemical analysis. In vitro results showed that BMSC-Exos-HIF1 $\alpha$ stimulated the proliferation of BMSCs and upregulated the expression level of bone-related genes, which was superior to bone marrow MSC exosomes (BMSC-Exos). In vivo results showed that BMSC-Exos-HIF1 $\alpha$ combined with $\beta$-TCP scaffold promoted new bone regeneration and neovascularization in the bone defect area, and the effect was better than that of BMSC-Exos combined with $\beta$-TCP scaffold. In this study, the results showed that BMSC-Exos-HIF1 $\alpha$ stimulated the proliferation and osteogenic differentiation of BMSCs and that BMSC-Exos-HIF1 $\alpha$ combined with $\beta$-TCP scaffolds could repair critical-sized bone defects by promoting new bone regeneration and neovascularization. 


\section{INTRODUCTION}

Repair and reconstruction of critical-sized bone defects caused by trauma, bone tumor, infection, etc. has always been a difficult task in the field of orthopedic treatment (Zhao et al., 2015). Current clinical methods of repairing bone defects include autologous bone grafting, allogeneic bone grafting, and combination of scaffold material with growth factors or cells. Autologous bone grafting is often considered the gold standard for repairing bone defects, but its use has a number of limitations, including a high incidence of donor sites, limited availability, relatively high and unpredictable autologous absorption (Benic and Hämmerle, 2014). Homozygous allogeneic bone grafting limits its potential clinical application due to high susceptibility to immune rejection (Behrend et al., 2016). With the development of bone tissue engineering, the development of scaffold materials has made great progress, beta-tricalcium phosphate $(\beta$-TCP) has good bioactivity, biodegradability, biocompatibility and is an ideal material for bone tissue repair and replacement (Gao et al., 2014; Oryan and Alidadi, 2018), but it doesn't exactly mimic the physiological function of bone tissue.

Hypoxia-inducible factor- $1 \alpha$ (HIF- $1 \alpha)$ plays an important role in the repair of bone defects (Yellowley and Genetos, 2019). On the one hand, HIF- $1 \alpha$ promotes the differentiation of bone marrow mesenchymal stem cells (BMSCs) to osteogenic cells and enhances osteogenic capacity (Costa et al., 2017). On the other hand, HIF- $1 \alpha$ mobilizes endothelial progenitor cells (EPCs) through the CXCL12/CXCR4 biotaxis to homing to the ischemic site and promotes microvascular regeneration at the bone defect site (Bianco et al., 2015; Kawakami et al., 2015; Fu et al., 2018; Ju et al., 2018; Zhao et al., 2018). Good blood supply is a prerequisite for bone tissue regeneration (Zhang et al., 2017). Therefore, we hope to repair the critical-sized bone defect by some way to make HIF$1 \alpha$ continuously expressed at the site of the bone defect through the dual role of HIF-1 $\alpha$ in promoting osteogenesis and angiogenesis.

However, the physiologically expressed HIF- $1 \alpha$ is degraded within minutes under normoxic conditions, and the preparation of active HIF- $1 \alpha$ that is not easily degraded under normoxic conditions is a key issue that needs to be addressed. We have previously genetically engineered three amino acid loci of the HIF- $1 \alpha$ subunit functional region (CDS region) to mutate to alanine at 402, 564, and 803, and confirmed that mutant HIF$1 \alpha$ can effectively maintain cellular expression under normoxic conditions, further in vivo tests have found that mutant HIF- $1 \alpha$ modified bone marrow mesenchymal stem cells (BMSC-HIF1 $\alpha$ ) effectively promote angiogenesis and have not found this graft to cause tumorigenicity in experimental animals (Qi et al., 2013; Yang et al., 2014).

In recent years, studies have shown that the role of stem cells in tissue repair is accomplished through the activation of peripheral receptor cells by exosomes secreted by their paracrine mechanism (Tan et al., 2020; Wang et al., 2020; Zhang B. et al., 2020). The exosome molecule is $50-150 \mathrm{~nm}$ in diameter, and it mainly contains cytokines, growth factors and other proteins, lipids, coding, or non-coding RNAs similar to the cells of origin, and participates in cell communication, cell migration, vascular regeneration and other processes, which plays an important role in the regulation of cell physiological functions ( $\mathrm{Li}$ et al., 2018). Application of exosomes in heterozygous animals does not induce a significant immune response (Roccaro et al., 2013). The application of BMSC-exosomes carrying mutant HIF1 $\alpha$ (BMSC-Exos-HIF1 $\alpha$ ) to repair critical-sized bone defects has not yet been reported.

The purpose of this study is to evaluate the role of BMSC-Exos-HIF1 $\alpha$ in repairing critical-sized bone defects. Our results show for the first time that BMSC-Exos-HIF1 $\alpha$ can effectively stimulate the proliferation and osteogenic differentiation of BMSCs, and the effect is better than that of BMSC-Exos; BMSC-Exos-HIF1 $\alpha$ combined with $\beta$-TCP scaffold material can significantly promote new bone formation and neovascularization in cranial critical-sized bone defects in rats' with better results than BMSC-Exos combined with $\beta$-TCP scaffold. BMSC-Exos-HIF1 $\alpha$ is expected to be an effective treatment for critical-sized bone defects in the clinic.

\section{MATERIALS AND METHODS}

This experiment was approved by the Ethics Committee of the Shanghai General Hospital, Shanghai Jiao Tong University School of Medicine. We have previously prepared mutant BMSCHIF1 $\alpha$ and related research papers have been published (Qi et al., 2013; Yang et al., 2014). The BMSC-HIF1 $\alpha$ used in this experiment was provided by Professor Danping Liu's team at Jinzhou Medical University.

\section{Isolation and Identification of BMSC-Exos-HIF1 $\alpha$}

When BMSC-HIF1 $\alpha$ reached $80-90 \%$ confluence, the medium was replaced with MGro-500 serum-free MSC medium (StemRD) and the cells were incubated for an additional $48 \mathrm{~h}$. Conditioned medium from BMSC-HIF1 $\alpha$ was collected and obtained by centrifugation at $3,00 \times g$ for $10 \mathrm{~min}$ and $2,000 \times g$ for $10 \mathrm{~min}$, respectively. The supernatant was filtered in a Steritop ${ }^{\mathrm{TM}} 0.22 \mu \mathrm{m}$ filter sterilizer (Millipore, Billerica MA, United States), followed by ultrafiltration in a $15 \mathrm{~mL}$ centrifugal filter test tube (Millipore), and finally the supernatant was centrifuged at $4,000 \times \mathrm{g}$ to approximately $200 \mu \mathrm{L}$ of liquid, the obtained suspension was washed twice with $15 \mathrm{~mL}$ PBS and ultrafiltered again with $4,000 \times g$ to $200 \mu \mathrm{L}$. To purify the exosomes, the suspension was covered with $30 \%$ sucrose- $\mathrm{D}_{2} \mathrm{O}$ pads in sterile Ultra-Clear ${ }^{\mathrm{TM}}$ tubes (Beckman Coulter, Brea, CA, United States) and ultracentrifuged at $1,00,000 \times g$ for $2 \mathrm{~h}$, the precipitated vesicles were thought to be exosomes. The precipitated exudate weight was suspended in $15 \mathrm{~mL}$ PBS and centrifuged at 4,000 $\times g$ in a centrifugal filtration test tube until the final volume was reduced to approximately $200 \mu \mathrm{L}$. All procedures were performed at $4^{\circ} \mathrm{C}$. Tunable resistive pulse sensing analysis and western blotting for the identification of BMSC-ExosHIF1 $\alpha$, data analysis using Izon Control Suite software v2.2 (Izon Science). 


\section{Extraction and Culture of Rat Bone Marrow Mesenchymal Stem Cells}

The experimental animals were 250-300 g Sprague-Dawley (SD) rats, Bone marrow from the humerus and femur of eight rats. Cells from rats were inoculated on a $10 \mathrm{~cm}^{2}$ plastic tissue culture plate containing $10 \%$ fetal bovine serum, $100 \mathrm{IU} / \mathrm{mL}$ penicillin, and $100 \mu \mathrm{g} / \mathrm{mL}$ streptomycin in MEM medium (MEM- $\alpha$; Invitrogen, Carlsbad, CA, United States), incubated at $37^{\circ} \mathrm{C}$ in an incubator containing $5 \% \mathrm{CO} 2$ for $48 \mathrm{~h}$. Nonadherent cells were discarded and defined as first generation cells (P1) when they grew to $80 \%$ confluence, P3 cells were used for all experiments.

\section{Cell Proliferation Assay}

The effect of BMSC-Exos-HIF1 $\alpha$ on BMSCs proliferation was detected by Cell Count Kit-8 (CCK-8; Dojindo). Cells were randomly divided into three groups: control group (adding the same amount of cell medium); experimental control group (adding BMSC-Exos to $200 \mu \mathrm{g} / \mathrm{mL}$ concentration); experimental group (adding BMSC-Exos-HIF1 $\alpha$ to $200 \mu \mathrm{g} / \mathrm{mL}$ concentration). $3 \times 10^{3}$ BMSCs ( $\mathrm{p} 3$ ) were grown in 96-well plates and enriched for $48 \mathrm{~h}$. BMSC-Exos-HIF1 $\alpha$ were then added to 96-well plates and incubated for an additional 1, 3, and 5 days, with the BMSC-Exos group performing the same operation. At the end of the incubation period, cells were incubated with $10 \mu \mathrm{L}$ CCK-8 solution for approximately $4 \mathrm{~h}$. Cell proliferation was calculated by measuring absorbance at $450 \mathrm{~nm}$ using an enzyme standardizer (BIO-TEK, ELX 800). The cell proliferation assay took three replicate wells for each group, and the experiment was repeated three times.

\section{Alkaline Phosphatase Activity Assay and Alizarin Red Staining}

Three groups of cells were inoculated in 6-well plates and the destination cells were stimulated by three different methods, cultured in freshly formed osteogenic medium (OM) for 14 days, and ALP activity was determined using an ALP assay kit (Beyotime Institute of Biotechnology, Shanghai, China), the experiment was repeated three times with three replicate wells each group. Alizarin Red staining (Sigma, St Louis, MO, United States) was used to detect bone mineralization. The cells of each group were incubated with the corresponding experimental stimuli for 21 days, the supernatant was discarded, washed twice with PBS, fixed with $4 \%$ paraformaldehyde for 15 min, discarded, washed twice with PBS again, stained with $2 \%$ cisplatin red, stained at room temperature for $20 \mathrm{~min}$, bone mineralization of specimens was observed by phase-contrast microscopy after the staining.

\section{Real-Time Quantitative Polymerase Chain Reaction}

The effect of BMSC-Exos-HIF1 $\alpha$ on the osteogenic differentiation of BMSCs was measured by Real-Time Quantitative Polymerase Chain Reaction (RT-qPCR) and all samples were tested in triplicate. Total RNA was extracted from three groups of cells by Trizol methods on day 3 and 7 ,
TABLE 1 | Primer sequences used in RT-qPCR.

\begin{tabular}{ll}
\hline Molecule & Primer sequences \\
\hline RUNX-2 & CCGAGCTACGAAATGCCTCT \\
& GGACCGTCCACTGTCACTTT \\
ALP & GCTTCTGTTCTGTAAGACGGG \\
& ACATGTTCAGCTTTGTGGACC \\
COL1-A1 & AGGTTTCCACGTCTCACCAT \\
\hline
\end{tabular}

respectively, RNA was reverse transcribed using the PrimeScript RT reagent kit, mRNA was reverse transcribed to CDNA and amplified by using the SYBR kit (TakaRa Bio, Shiga, Japan). $\beta$-actin as a control. The required primer information is listed in Table 1.

\section{Model of Cranial Deficiency in Rats}

Thirty nine mature Sprague Dawley rats (12 weeks old, mean weight 250-300 g) were used for in vivo experimental studies. All surgical procedures are performed under general anesthesia and postoperative analgesic care with tramadol is ensured. All efforts are made to minimize the suffering of the animal. All operations are carried out under sterile conditions. We used classically porous $\beta$-TCP scaffolds with an average pore size of $500 \mu \mathrm{m}$ and a classically porous $\beta$-TCP scaffold with $75 \%$ porosity (the subscaffold material has a diameter of $5 \mathrm{~mm}$ and a height of $2 \mathrm{~mm}$ ) as BMSC-Exos and BMSC-Exos-HIF1 $\alpha$ carriers. $200 \mu \mathrm{g}$ BMSC-Exos and $200 \mu \mathrm{g}$ BMSC-Exos-HIF1 $\alpha$ drops were added to a sterile $\beta$-TCP holder under sterile conditions and freezedried for at least $4 \mathrm{~h}$. Application of $4 \%$ hydrated chloral by intraperitoneal injection to anesthetize 30 rats, after successful anesthesia, the rats were placed prone on a fixed plate, head shaving, sterilized with iodine and spread sterile towels. A $1.5 \mathrm{~cm}$ sagittal incision is made in the scalp, the fascia is cut, the periosteum is peeled off the surface of the skull to both sides with a small bone peel, then a $5 \mathrm{~mm}$ diameter dental ring drill is used to drill a $5 \mathrm{~mm}$ diameter full-layer skull defect on each side of the skull surface on the median line (injury to the dura mater and brain tissue is strictly prohibited during surgery). The rats were randomly divided into three groups: $\beta$-TCP group (13): $\beta$-TCP stent only; $200 \mu \mathrm{g}$ BMSC-Exos group $+\beta$-TCP (13): $200 \mu \mathrm{g}$ BMSC-Exos $+\beta$-TCP stent; $200 \mu \mathrm{g}$ BMSC-Exos-HIF1 $\alpha$ group $+\beta$-TCP (13): $200 \mu \mathrm{g}$ BMSC-Exos-HIF1 $\alpha+\beta$-TCP stent. Suture the surgical incision layer by layer after implanting the test material into the defect in each group. For three days after surgery, 40,000 units of gentamicin were injected daily to prevent infection. At 12 weeks postoperatively, the skulls of all experimental rats were removed after death by overanesthesia and stored in a $4 \%$ paraformaldehyde solution buffered in $0.1 \mathrm{M}$ phosphate solution ( $\mathrm{pH}$ 7.2).

\section{Micro-CT Analysis}

Analysis of data related to bone defect areas using a Micro CT system (mCT-80, Scanco Medical, Brüttisellen, Switzerland). The fixed specimens were subjected to Micro-CT (SKYSCAN 1176 , Bruker) with the following parameters: operating voltage 
$80 \mathrm{KV}$, exposure time $240 \mathrm{~ms}$, thickness of the specimen scanned $18 \mu \mathrm{m}$. Skyscan software is included in the micro-CT system used in this experiment and Skyscan software includes CT-An, CT-Vol, CT-Vox and other applications. The 2D images were obtained directly after Micro-CT scan, the $3 \mathrm{D}$ reconstruction images were constructed using CT-Vol application, and the bone mineral density (BMD) and bone volume fraction (BV/TV) were automatically determined by using CT-An application. BMD and $\mathrm{BV} / \mathrm{TV}$ values in the bone defect area were assessed for new bone formation by using auxiliary software (Scanco), each group randomly selected 6 samples for analysis and detection.

\section{Sequential Fluorescent Labeling}

Intraperitoneal injections of tetracycline hydrochloride (TE, $25 \mathrm{mg} / \mathrm{kg} \mathrm{bw}$ ), alizarin red (AL, $30 \mathrm{mg} / \mathrm{kg} \mathrm{bw}$ ) and calcitonin $(\mathrm{CA}, 20 \mathrm{mg} / \mathrm{kg} \mathrm{bw})$ were given to rats at 2, 4, and 6 weeks postoperatively, respectively. Application of confocal laser scanning microscopy (CLSM, Leica) to examine sections for data analysis and image acquisition by three different fluorescence reactions. Yellow fluorescence images indicate TE, red fluorescence images indicate $\mathrm{AL}$, and green fluorescence images indicate CA, indicating new bone formation and new bone tissue mineralization at weeks 2, 4, and 6 after surgery, respectively. Percentages of three staining results for TE, AL and CA were analyzed by histomorphometric analysis, each group randomly selected six samples for analysis and detection.

\section{Microfil Perfusion Testing}

Rats killed by chloral hydrate overanesthesia were perfused with Microfil infusion (Flowtech, Carver, MA, United States). A longitudinal incision was made in the rat's chest to open the descending aorta, a vascular catheter was placed in the left ventricle, the inferior vena cava was cut, $20 \mathrm{~mL}$ of heparinized saline was repeatedly perfused, then $20 \mathrm{~mL}$ of Microfil was perfused at $2 \mathrm{~mL} / \mathrm{min}$, and the vessel was ligated with sutures after perfusion, and the rat was kept in a $4^{\circ} \mathrm{C}$ refrigerator overnight. The cranial specimens were kept in formalin solution for more than $24 \mathrm{~h}$, decalcified in decalcification solution for about 2 weeks, and then the specimens were scanned under the Micro CT system and the neovascularization in the cranial defect was represented by $3 \mathrm{D}$ reconstruction image. The area and number of local blood vessels in bone defects were evaluated by CT-Vox program, each group randomly selected 6 samples for analysis and detection.

\section{Histomorphology and \\ Immunohistochemistry Analysis}

Six skull samples were randomly selected from each group and dehydrated through a concentration gradient from 70 to $100 \%$ ethanol and then embedded in polymethyl methacrylate (PMMA). The cranial specimens were then cut into longitudinal sections 150-200 $\mu \mathrm{m}$ thick using a hard tissue biopsy machine (Leica Microsystems, Wetzlar, Germany), glued onto plastic carrier slides and sandpapered thin and polished to a final thickness of about $50 \mu \mathrm{m}$. Sections were fluorescently labeled with laser confocal scanning microscopy (Leica) to quantify new bone formation and bone mineralization at four locations, and the average of four measurements was calculated to assess the mean values for each group. Sections were stained with van Gieson's to evaluate new bone formation, with red areas representing new bone formation and black areas representing $\beta$-TCP stents. Three sections were randomly selected from each skull samples, with 18 slices in each group, a total of 54 slices were used for analysis of new bone formation. Image acquisition for quantitative evaluation of the area of new bone formation by Image J software. Another portion of each cranial specimen was decalcified in a decalcification solution for approximately two weeks, dehydrated by gradient ethanol, encased in paraffin, cut into $5 \mu \mathrm{m}$ thick sections, and immunohistochemically analyzed for osteocalcin (OCN) and CD31 to assess bone formation and angiogenesis, respectively.

\section{Statistical Analysis}

Statistical analysis was performed using SPSS 22.0 software (Release 22.0; SPSS, Chicago, IL, United States). All of the data were shown as the mean \pm standard deviation (SD). The Kolmogorov-Smirnov test was used to analyze whether the data obeyed a normal distribution. The test of homogeneity of variance was used to analyze whether the data met the homogeneity of variance. For the data with normal distribution, the difference between groups was calculated by one-way analysis of variance (ANOVA), otherwise, non-parametric test was used. The difference was significant when $P$-value $<0.05$.

\section{RESULTS}

\section{Identification of BMSC-Exos-HIF1 $\alpha$}

Identification of BMSC-Exos-HIF1 $\alpha$ using Tunable Resistive Pulse Sensing (TRPS) analysis and Western blotting. The results showed that most of the particles in the sample were distributed in the range of $50-150 \mathrm{~nm}$ (Figure 1A), and the experimental results showed that they belonged to the BMSCExos-HIF $1 \alpha$. Western blotting results showed that these particles express characteristic surface markers (CD9, CD63, and CD81) (Figure 1B) and HIF-1 $\alpha$ (Figure 1C), which further confirmed that they are BMSC-Exos-HIF1 $\alpha$.

\section{BMSC-Exos-HIF1 $\alpha$ Promotes Proliferation and Osteogenic Differentiation of BMSCs}

The CCK-8 method was used to evaluate the effect of BMSC-Exos-HIF1 $\alpha$ on BMSCs proliferation (Figure 2C). The experimental results showed a statistically significant difference between the $200 \mu \mathrm{g} / \mathrm{mL}$ BMSC-Exos-HIF1 $\alpha$ group and the control, $200 \mu \mathrm{g} / \mathrm{mL}$ BMSC-Exos group $(P<0.05)$. The results showed that BMSC-Exos-HIF1 $\alpha$ was effective in promoting the proliferation of BMSCs and was more effective than BMSC-Exos. ALP activity as an early marker of osteogenic differentiation, the results of this experiment were shown in Figures 2A1-A3, more pronounced ALP staining was observed in cells treated with $200 \mu \mathrm{g} / \mathrm{mL}$ BMSC-Exos-HIF1 $\alpha$. Quantitative analysis of 


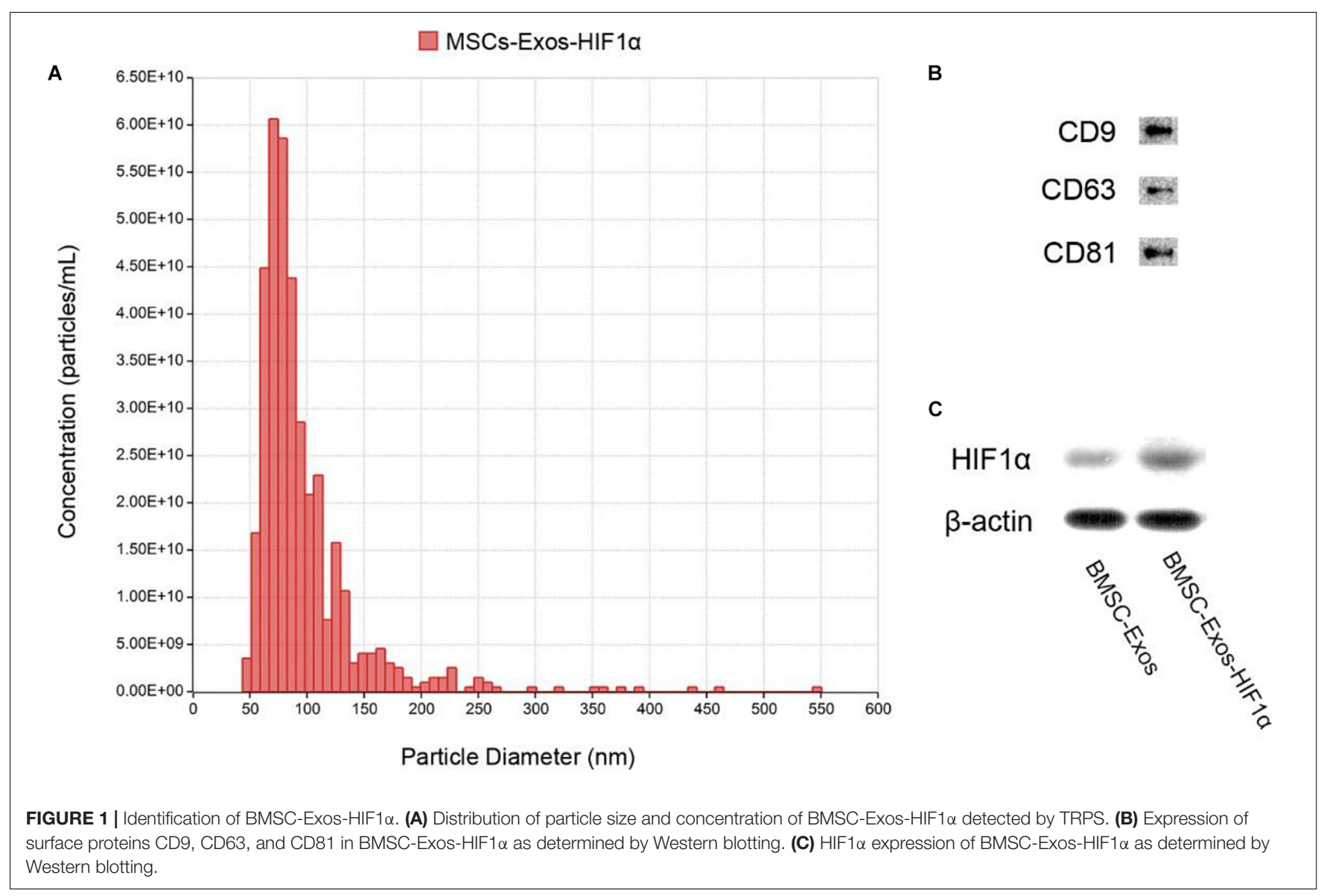

ALP activity (Figure 2D) showed a statistically significant difference $(P<0.05)$ between the $200 \mu \mathrm{g} / \mathrm{mL}$ BMSC-ExosHIF1 $\alpha$ group and the control, $200 \mu \mathrm{g} / \mathrm{mL}$ BMSC-Exos group at day 14 of culture. Alizarin red staining showed significantly enhanced mineral deposition in the $200 \mu \mathrm{g} / \mathrm{mL}$ BMSC-ExosHIF1 $\alpha$ group compared to the control and $200 \mu \mathrm{g} / \mathrm{mL}$ BMSCExos group (Figures 2B1-B3). Both ALP activity and alizarin red staining showed that BMSC-Exos-HIF1 $\alpha$ can effectively promote osteogenic differentiation of BMSCs, and the effect is better than BMSC-Exos. RT-qPCR assay showed that mRNA expression of RUNX-2, ALP and COL1 was significantly increased in cells treated with BMSC-Exos-HIF1 $\alpha$, and there was a statistically significant difference between the $200 \mu \mathrm{g} / \mathrm{mL}$ BMSC-ExosHIF1 $\alpha$ group and the control, $200 \mu \mathrm{g} / \mathrm{mL}$ BMSC-Exos group $(P<0.05)$. RT-qPCR results showed that BMSC-Exos-HIF1 $\alpha$ can up-regulate the expression of osteogenesis-related genes in BMSCs with better effect than BMSC-Exos (Figures 2E-G). In summary, in vitro studies show that BMSC-Exos-HIF1 $\alpha$ can effectively stimulate the proliferation of BMSCs and enhance their osteogenic differentiation, which is superior to BMSC-Exos.

\section{Micro-CT Analysis of New Osteogenesis in the Cranial Defect Area}

Micro-CT 3D reconstruction image analysis showing morphology of newly formed bone (Figures 3A1-C1, 3A2-C2).
In the sagittal view (Figures 3A3-C3), more newly formed bone can be observed in the $200 \mu \mathrm{g}$ BMSC-Exos-HIF1 $\alpha+\beta$-TCP group than in the $200 \mu \mathrm{gBMSC}$-Exos $+\beta$-TCP group or $\beta$-TCP group. Quantitative analysis showed that the BMD of the bone defect area was significantly higher in the $200 \mu \mathrm{gBMSC}$-ExosHIF $1 \alpha+\beta$-TCP group $\left(0.575 \pm 0.043 \mathrm{~g} / \mathrm{cm}^{3}\right)$ than in the $200 \mu$ gBMSC-Exos $+\beta$-TCP group $\left(0.406 \pm 0.041 \mathrm{~g} / \mathrm{cm}^{3}\right)$ or $\beta$-TCP group $\left(0.064 \pm 0.016 \mathrm{~g} / \mathrm{cm}^{3}\right)(P<0.05)$ (Figure $\left.3 \mathrm{D}\right)$. $\mathrm{BV} / \mathrm{TV}$ in the bone defect area was significantly higher in the $200 \mu$ gBMSC-Exos-HIF $1 \alpha+\beta$-TCP group $(38.03 \pm 3.08 \%)$ than in the $200 \mu$ gBMSC-Exos $+\beta$-TCP group $(23.51 \pm 3.04 \%)$ or $\beta$-TCP group $(4.46 \pm 1.42 \%)(P<0.05)$ (Figure 4E). The above results indicate that BMSC-Exos-HIF $1 \alpha+\beta$-TCP can effectively promote new bone regeneration, and the effect is better than BMSC-Exos $+\beta$-TCP.

\section{Fluorochrome Labeling Histomorphometric Analysis}

Figure 4 shows the results of new bone formation and mineralization by fluorescence labeling analysis at weeks 2, 4, and 6 post-modeling. At week 2, the percentage of TE markers (yellow) in the $200 \mu \mathrm{g}$ BMSC-ExosHIF $1 \alpha+\beta$-TCP group $(4.21 \pm 0.23 \%)$ was significantly higher than in the $200 \mu \mathrm{g}$ BMSC-Exos $+\beta$-TCP group $(3.11 \pm 0.17 \%)$ or $\beta$-TCP group $(0.34 \pm 0.09 \%)(P<0.05)$ 


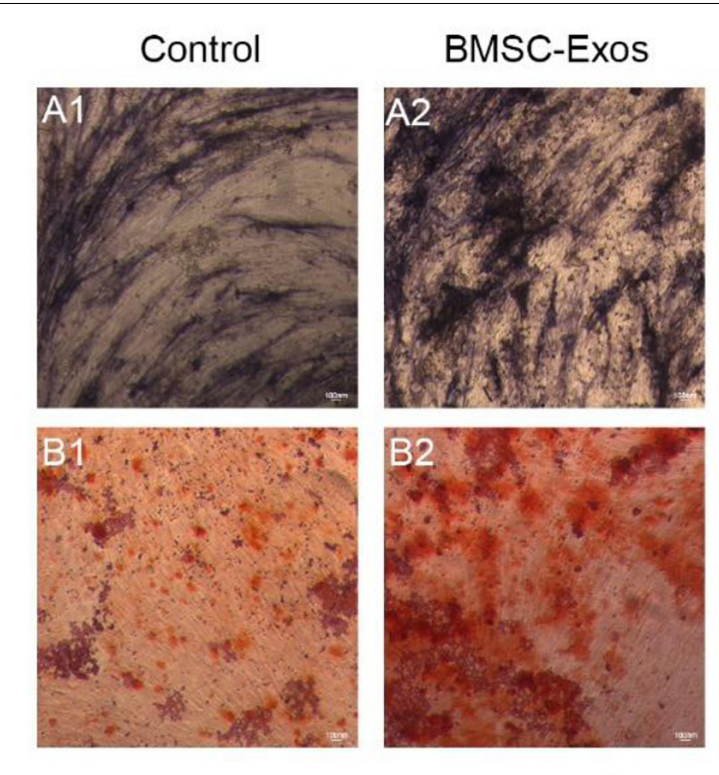

\section{BMSC-Exos-HIF1a}
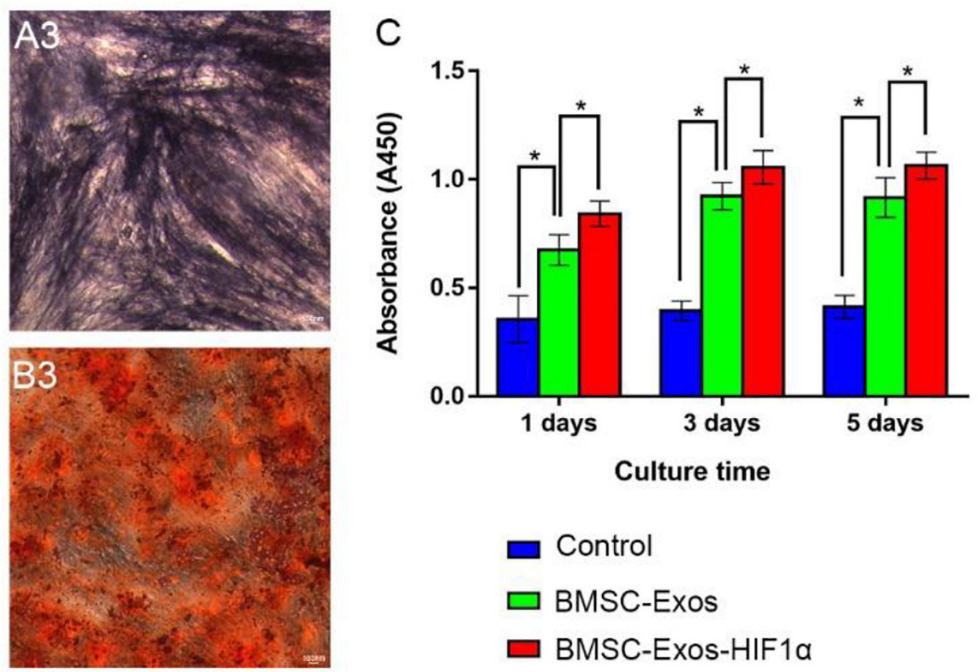

- Control

$\square$ BMSC-Exos

$\square$ BMSC-Exos-HIF1a
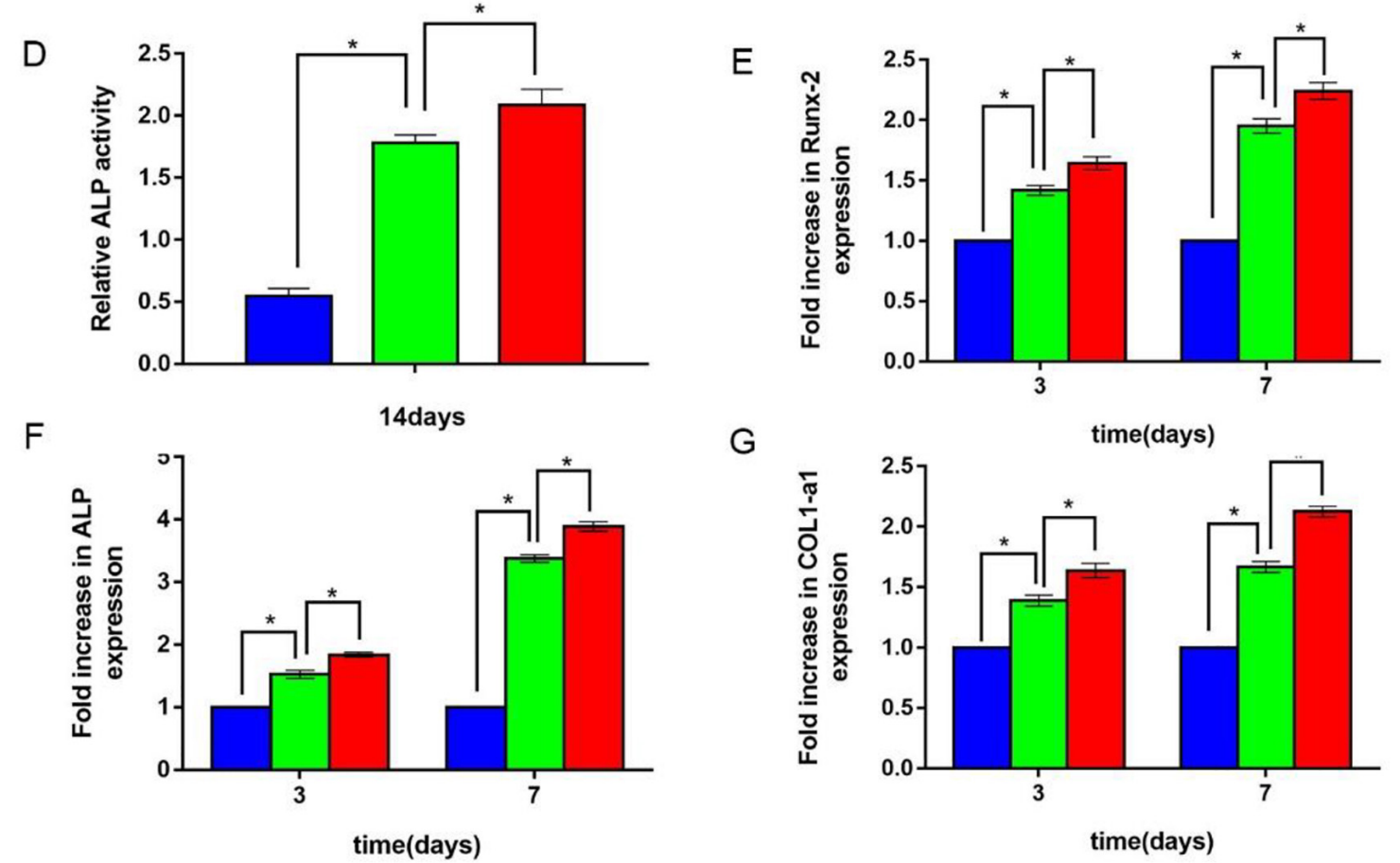

FIGURE 2 | BMSC-Exos-HIF1 $\alpha$ promotes proliferation and osteogenic differentiation in bone marrow mesenchymal stem cells. Alkaline phosphatase activity assay (14 days) (A1-A3) and quantitative analysis (D), Alizarin red staining (21 days) (B1-B3) to determine the effect of BMSC-Exos-HIF1 $\alpha$ on osteogenic differentiation of BMSCs. (C) CCK-8 method to assess the effect of BMSC-Exos-HIF1 $\alpha$ on the proliferation of BMSCs. RT-qPCR method to detect gene expression levels of RUNX-2 (E), ALP (F), and COL1 (G) on the third and seventh day of culture. All tests were repeated three times ( ${ }^{*}$ indicates significant difference, $P<0.05$; scale bar: $100 \mu \mathrm{m})$.

(Figures 4A1-C1). At week 4, the percentage of AL markers (red) in the $200 \mu \mathrm{g}$ BMSC-Exos-HIF1 $\alpha+\beta$-TCP group $(4.32 \pm 0.26 \%)$ was significantly higher than in the $200 \mu \mathrm{g}$ BMSC-Exos $+\beta$-TCP group $(3.18 \pm 0.22 \%)$ or the $\beta$-TCP group $(0.38 \pm 0.08 \%)(P<0.05)$ (Figures 4A2-C2). At week 6 , the percentage of CA markers (green) in the $200 \mu$ gBMSC-Exos-HIF1 $\alpha+\beta$-TCP group $(4.39 \pm 0.27 \%)$ was significantly higher than in the $200 \mu \mathrm{g}$ BMSCExos $+\beta$-TCP group $(3.24 \pm 0.23 \%)$ or $\beta$-TCP group $(0.41 \pm 0.09 \%)(P<0.05)$ (Figures 4A3-C3). The above results indicate that BMSC-Exos-HIF1 $\alpha+\beta$-TCP can effectively promote the regeneration of new bone in the bone defect area and its effect is better than that of BMSC-Exos $+\beta$-TCP. 

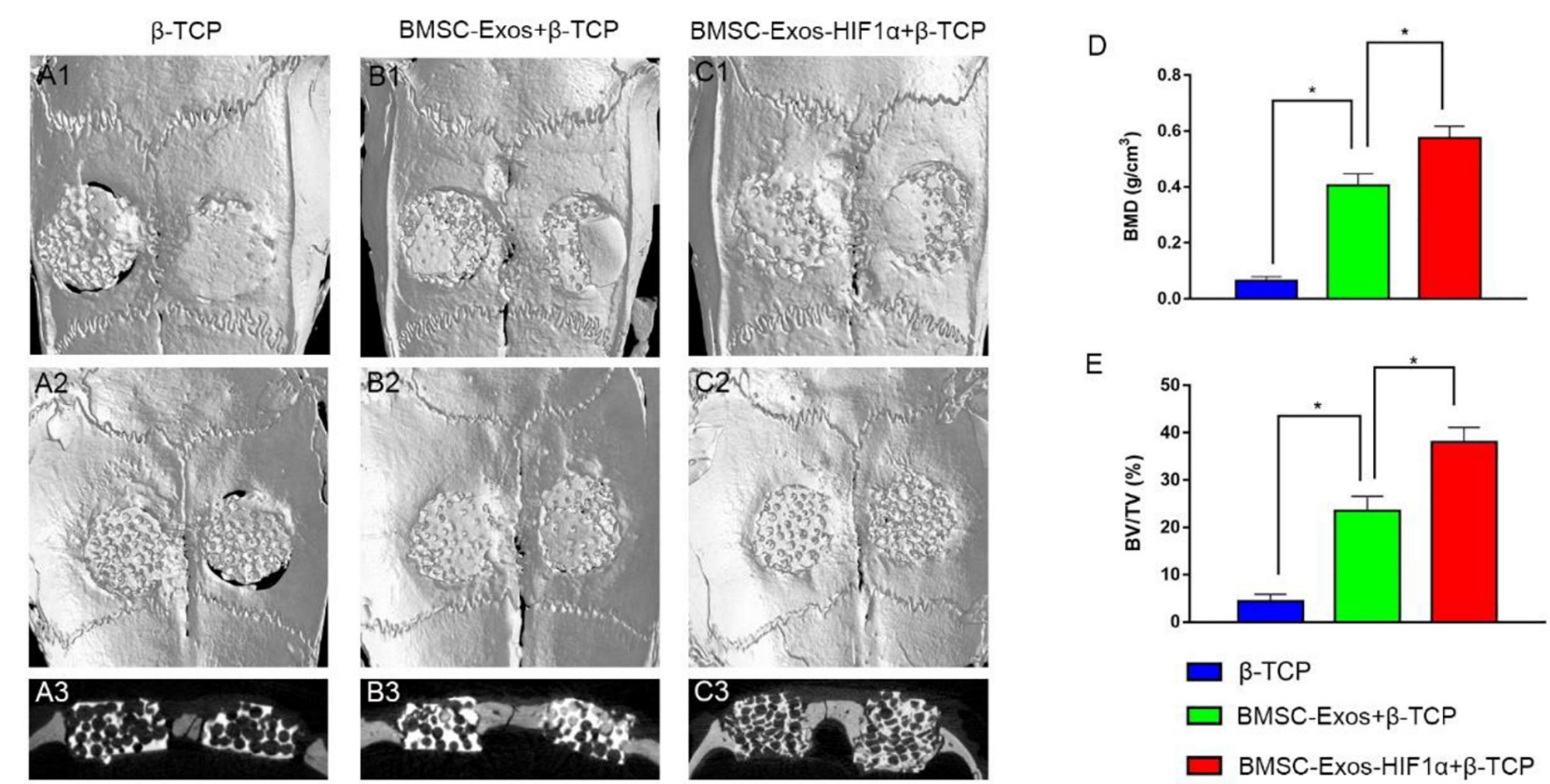

FIGURE 3 | Micro-CT analysis of BMSC-Exos-HIF1 $\alpha+\beta$-TCP promoting new bone regeneration. Analysis of three-dimensional Micro-CT images at 12 weeks post-modeling, external cranial reconstruction (A1-C1), internal cranial reconstruction (A2-C2), sagittal position (A3-C3); Quantitative analysis of bone mineral density (BMD) (D) and bone volume fraction (BV/TV) (E) at 12 weeks after surgery $\left(n=6,{ }^{*}\right.$ indicates statistically significant difference, $\left.P<0.05\right)$.

\section{Analysis of Neovascular Formation in Bone Defects}

Micro-CT image analysis showed that the $200 \mu \mathrm{gBMSC}$ Exos-HIF1 $\alpha+\beta$-TCP group had more neovascularization compared to the $200 \mu \mathrm{gBMSC}$-Exos $+\beta$-TCP group, $\beta$-TCP group (Figures 5A1-A3). Quantitative analysis of the area of neovascular formation (Figure 5B) and the number of neovessels (Figure 5C) showed the same results. There was a statistically significant difference in the number of neonatal vessels in the $200 \mu \mathrm{gBMSC}$-Exos-HIF1 $\alpha+\beta$-TCP group (76.8 \pm 3.4 ) compared to the $200 \mu \mathrm{gBMSC}$-Exos $+\beta$-TCP group $(63.9 \pm 3.8)$ and the $\beta$-TCP group $(6.8 \pm 2.4)(P<0.05)$. The $200 \mu$ gBMSC-Exos-HIF $1 \alpha+\beta$-TCP group had the largest area of neovascularization $(74.91 \pm 4.05 \%)$ and a statistically significant difference compared to the $200 \mu \mathrm{gBMSC}$-Exos $+\beta$-TCP group $(65.24 \pm 3.65 \%)$ and the $\beta$-TCP group $(3.38 \pm 0.98 \%)$ $(P<0.05)$. The experimental results showed that BMSC-ExosHIF $1 \alpha+\beta$-TCP can effectively promote neovascularization in the bone defect area, and the effect is better than BMSCExos $+\beta$-TCP.

\section{Histological and Immunohistochemical Analysis}

Analysis of the Van Gieson staining results of undecalcified samples showed a significant increase in new osteogenesis in the $200 \mu \mathrm{g}$ BMSC-Exos-HIF1 $\alpha+\beta$-TCP group (60.87 $\pm 3.71 \%)$, the area of new bone formation was significantly larger than $200 \mu \mathrm{g}$ BMSC-Exos $+\beta$-TCP group $(36.52 \pm 3.47 \%)$ and $\beta$-TCP group $(6.26 \pm 1.19 \%)(P<0.05)$, and there was a statistically significant difference between the three groups $(P<0.05)$ (Figure 6). The histological staining results showed the same trend as the results of the new bone regeneration Micro-CT analysis.

Immunohistochemical staining of decalcified skulls for the osteogenic marker $\mathrm{OCN}$ and the neovascularization marker CD31 (Figure 7), OCN results showed almost no significant positive staining in the $\beta$-TCP group (Figure 7A1), however, OCN-positive staining was evident in the $200 \mu \mathrm{gBMSC}$-Exos $+\beta$-TCP group (Figure 7A2) and the $200 \mu \mathrm{gBMSC}$-ExosHIF1 $\alpha+\beta$-TCP group (Figure 7A3). $200 \mu$ gBMSC-ExosHIF1 $\alpha+\beta$-TCP showed the most obvious positive staining results for OCN. Compared to the $\beta$-TCP group (Figure 7B1), the CD31 positive staining in the $200 \mu \mathrm{g}$ BMSC-ExosHIF $1 \alpha+\beta$-TCP group (Figure 7B2) or the $200 \mu \mathrm{g}$ BMSCExos-HIF1 $\alpha+\beta$-TCP group (Figure 7B3) was significant, the most pronounced $\mathrm{CD} 31$ positive staining was observed in the $200 \mu \mathrm{g}$ BMSC-Exos-HIF1 $\alpha+\beta$-TCP group. The results showed that BMSC-Exos-HIF $1 \alpha+\beta$-TCP can effectively promote the expression of osteogenic markers and vascular neovascularization markers in the bone defect area, and the effect was better than that of BMSC-Exos $+\beta$-TCP.

\section{DISCUSSION}

Due to the limited inherent regenerative capacity of bone, criticalsized bone defects caused by trauma, bone tumor removal, etc. are difficult to cure through autologous bone regeneration. How to repair critical-sized bone defects has always been a difficult task in orthopedics. Although autologous bone grafting is considered the 


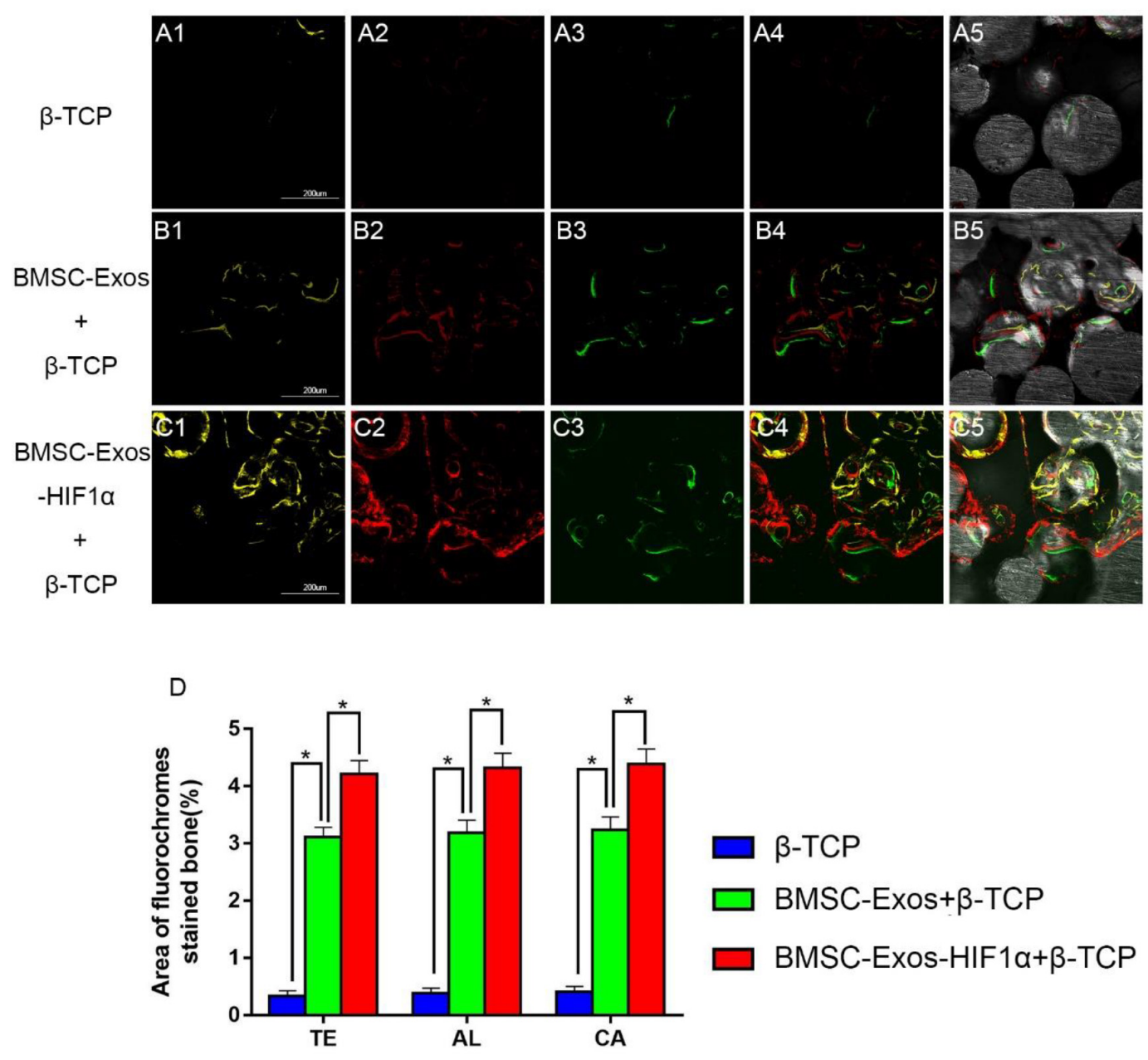

fluorochromes of bone markers

FIGURE 4 | Sequential fluorescent labeling analysis of BMSC-Exos-HIF1 $\alpha+\beta$-TCP to promote new bone regeneration. Yellow images (A1-C1) indicate TE, red images (A2-C2) indicate AL, and green images (A3-C3) indicate $\mathbf{C A}$, indicating new bone formation and mineralization at postoperative weeks 2, 4, and 6, respectively. Combined images of three fluorescent staining results (A4-C4), combined images of three fluorescent staining results with normal images (A5-C5), histomorphometric quantitative analysis of TE, AL, and CA fluorescence expression at 12 weeks after surgery (D). ( $n=6,{ }^{*}$ indicates statistically significant difference, $P<0.05$; scale bar: $200 \mu \mathrm{m})$.

gold standard for repairing bone defects, its application is limited by donor site morbidity, limited availability, and unpredictable autologous resorption, among other limitations (Rogers and Greene, 2012). Advances in bone tissue engineering provide a new avenue for bone defect repair, and tissue engineering strategies that use specific structures to repair bone defects have advantages over current bone grafting techniques (McGovern et al., 2018). Our results show for the first time that BMSC-ExosHIF1 $\alpha$ can effectively stimulate the proliferation and osteogenic differentiation of BMSCs and is more effective than BMSC-Exos, BMSC-Exos-HIF $1 \alpha+\beta$-TCP effectively promotes new bone regeneration and neovascularization in a rat cranial defect model, superior to BMSC-Exos $+\beta$-TCP.

In bone defect repair, angiogenesis and osteogenesis are closely related, with HIF-1 $\alpha$ playing an important role. HIF- $1 \alpha$ found to promote differentiation of BMSCs toward osteogenic cells
(Ding et al., 2013; Zhang J. et al., 2018); H1F-1 $\alpha$ and Runx2 interact via the Runt domain to upregulate VEGF expression to promote angiogenesis, and to upregulate Runx2 expression (Kwon et al., 2011; Lee et al., 2012). Runx2 is a transcription factor that plays an important regulatory role in bone formation and development and is essential for osteogenic cell differentiation, Runx2 as a target gene of the $\mathrm{Wnt} / \beta$-catenin signaling pathway activates the $\mathrm{Wnt} / \beta$-catenin signaling pathway to promote new bone formation (Lin et al., 2011; Cai et al., 2016; Thiagarajan et al., 2017; Vega et al., 2017). In addition, HIF-1 $\alpha$ was found to significantly upregulate CXCL12 expression at the ischemic site, prompting CXCL12 to diffuse to the periphery, forming a concentration gradient, ultimate promotion of mobilization and homing of EPCs via CXCL12/CXCR4 bio-axis enhances angiogenesis in the ischemic zone (Bianco et al., 2015; Kawakami et al., 2015). However, the physiologically expressed HIF-1 $\alpha$ 

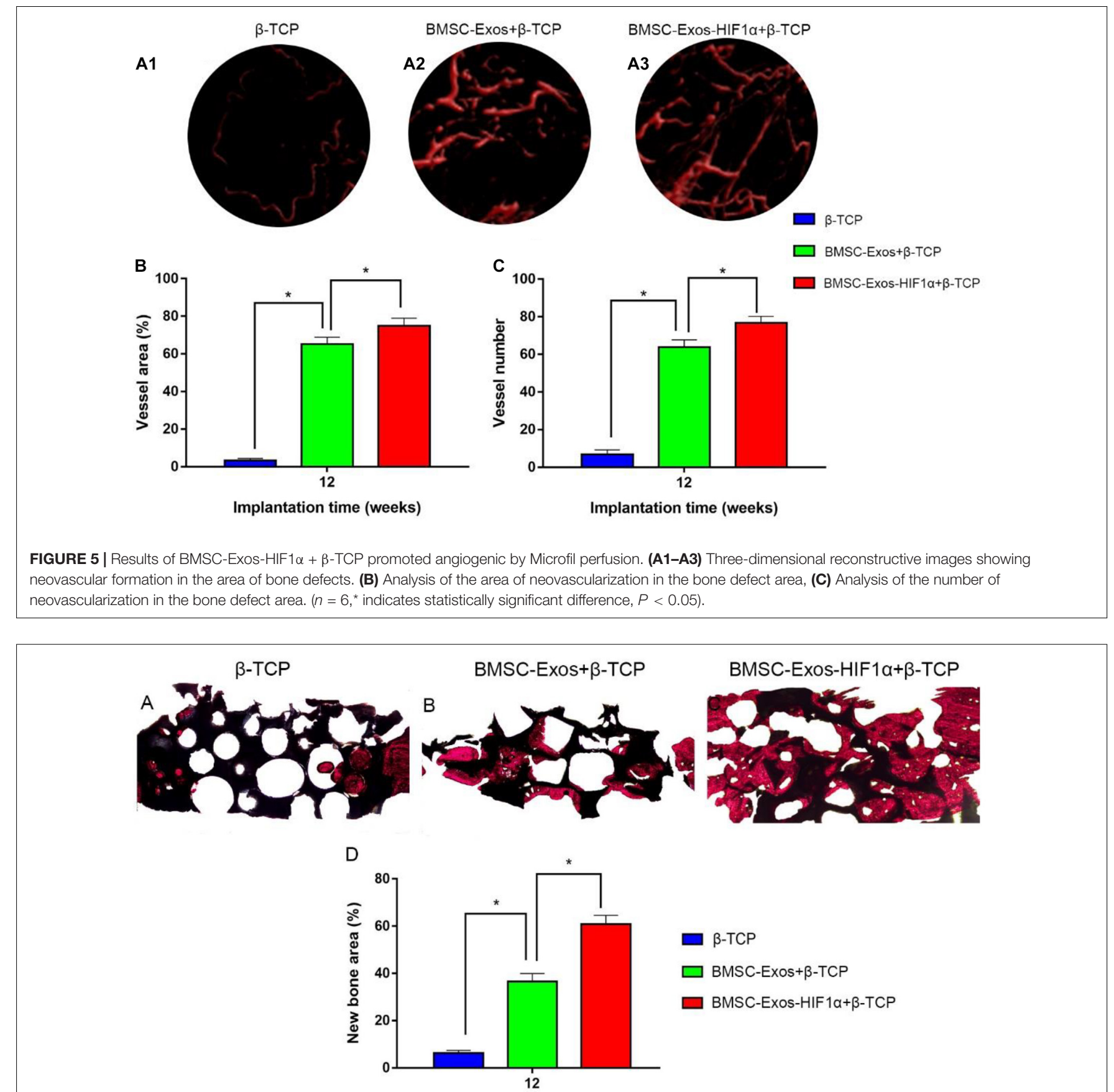

Implantation time (weeks)

FIGURE 6 | Histological analysis of BMSC-Exos-HIF1 $\alpha+\beta$-TCP scaffold promoting new bone regeneration. (A-C) Results of VG staining of hard tissue sections, red represents the area of new bone formation and black represents the part of the remaining scaffold material, (D) Results of quantitative histomorphological analysis 12 weeks after surgery $(X 40) .\left(n=6,{ }^{*}\right.$ indicates statistically significant difference, $\left.P<0.05\right)$.

is degraded by the body within minutes under normoxic conditions. It was found that the mechanism by which HIF-1 $\alpha$ is rapidly degraded by the organism under normoxic conditions is the HIF-1 $\alpha$ subunit functional region (CDS region) encoding 826 amino acids, which has an oxygen-dependent degradation structural domain (ODD) consisting of more than 200 amino acids, Hydroxylation of the 402nd and 564th proline residues in the ODD of the HIF- $1 \alpha$ molecule catalyzed by proline hydroxylase (oxygen is required for this hydroxylation process), and the hydroxylated HIF-1 $\alpha$ is rapidly degraded by ubiquitin protease (Jin et al., 2009; Kim et al., 2009; Wei et al., 2010). In contrast, under hypoxic conditions, because proline 402, 564 


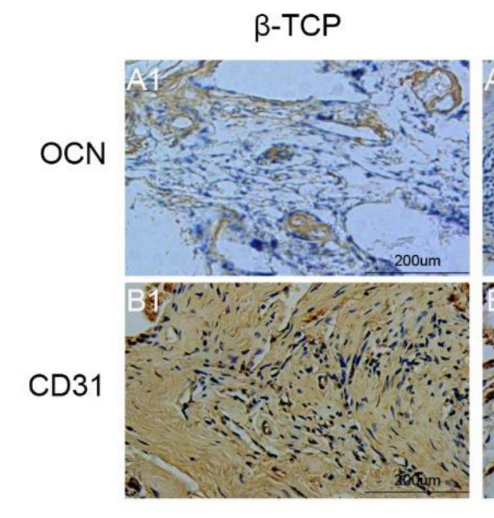

\section{BMSC-Exos $+\beta-$ TCP $\quad$ BMSC-Exos-HIF1 $\alpha+\beta-T C P$}
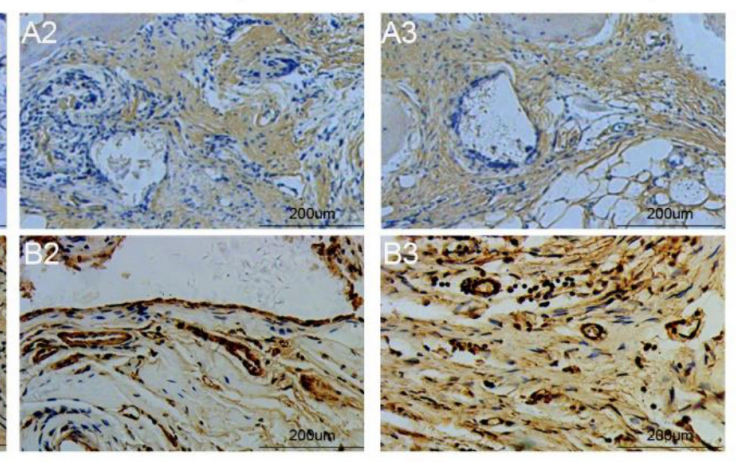

FIGURE 7 | Immunohistochemical analysis of BMSC-Exos-HIF1 $\alpha+\beta$-TCP for promoting new bone regeneration and neovascularization. Application of immunohistochemical analysis to detect new osteogenesis and neovascularization, OCN (A1-A3) and CD31 (B1-B3) in samples. (scale bar: 200 $\mu$ m).

cannot be hydroxylated, HIF- $1 \alpha$ accumulates and is transferred to the nucleus, where it activates the hypoxia-responsive gene cluster. It was also shown that the transcriptionally active region that determines HIF- $1 \alpha$ is located at position 803 asparagine at its carboxyl terminus (CAD) and that blocking its hydroxylation with chemical inhibitors or gene recombination leads to strong transcriptional activity of HIF- $1 \alpha$. Thus, the important structural regions of HIF- $1 \alpha$ include not only proline 402 and 564 in the ODD region, but also asparagine 803 in the CAD region. In this subject, 3-point mutant HIF-1 $\alpha$ was successfully prepared and BMSCs modified with 3-point mutant HIF-1 $\alpha$ were found to be effective in promoting vascularization in bone ischemic areas in vivo (Qi et al., 2013; Yang et al., 2014).

Bone marrow mesenchymal stem cells have superior osteogenic potential (Reinisch et al., 2015), can differentiate into osteoblasts and promote vascular regeneration through paracrine action (Phinney and Prockop, 2007; Satija et al., 2009; Wei et al., 2014), but the direct application of stem cells is limited by factors such as immune rejection, chromosomal variation, etc., (Wang et al., 2012). There are many components in exosomes (Phinney and Pittenger, 2017; Zhang S. et al., 2018), exosomes act as important participants in intercellular signaling by transferring functional proteins, RNA, microRNA and lncRNA between cells and can significantly reduce immune response (Poe and Knowlton, 2018; Zhao et al., 2020). The biological function of stem cells is mainly achieved by stimulating effector cells with specific biologically active molecules carried in the exocrine body. Studies have shown that BMSC-Exos can promote bone regeneration by promoting osteogenesis and angiogenesis (Nakamura et al., 2015; Xue et al., 2018; Takeuchi et al., 2019; Lee et al., 2020; Xu et al., 2020; Zhang L. et al., 2020). Therefore, our study aimed to investigate the effect of BMSC-Exos-HIF1 $\alpha$ on the proliferation and osteogenic differentiation of BMSCs, investigating the biological function of BMSC-Exos-HIF1 $\alpha$ loading on $\beta$-TCP scaffold material to repair bone defects in rats. However, our study also had some limitations that we did not explore the signal pathways and molecular mechanisms of BMSCExos-HIF1 $\alpha$ in repairing bone defects through promoting osteogenesis and angiogenesis, which will be further explored in our follow-up studies.

Assessment of the biological function of BMSC-Exos-HIF1 $\alpha$ to repair bone defects using a rat skull defect model. Micro-CT quantitative analysis showed that the application of BMSC-ExosHIF $1 \alpha+\beta$-TCP scaffold significantly increased the formation of new bone in the skull defect area of rats, and the amount of new bone formation was more than that of BMSCExos $+\beta$-TCP. Histological analysis showed that the $\beta$-TCP group showed almost no new bone formation in the area of bone defects, while both the BMSC-Exos $+\beta$-TCP group and the BMSC-Exos-HIF1 $\alpha+\beta$-TCP group showed the greatest amount of new bone formation, Histological analysis results are consistent with Micro-CT quantitative analysis. Microfil perfusion is used to evaluate the neovascularization of bone defect area in this experiment, analysis of both the area of neovascularization and the number of neovessels showed more neovascularization in both the BMSC-Exos $+\beta$-TCP group and the BMSC-Exos-HIF1 $\alpha+\beta$-TCP group, the BMSC-ExosHIF $1 \alpha+\beta$-TCP group showed the most neovascular formation. OCN and CD31 are common markers in studies of osteogenic differentiation and neovascularization, respectively. Analysis of immunohistochemical results showed that OCN and CD31 had only a small amount of positive staining expression in the bone defect area in the $\beta$-TCP group, and more positive staining results in the BMSC-Exos-HIF $1 \alpha+\beta$-TCP group than in the BMSC-Exos $+\beta$-TCP group.

In summary, BMSC-Exos-HIF1 $\alpha+\beta$-TCP could significantly repair critical-sized bone defects by enhancing neogenesis and neovascular formation in vivo.

\section{CONCLUSION}

In our current study, we demonstrate for the first time that BMSC-Exos-HIF1 $\alpha$ effectively stimulates the proliferation and osteogenic differentiation of BMSCs, and is more effective than BMSC-Exos. BMSC-Exos-HIF1 $\alpha$ combined with $\beta$-TCP scaffolds is superior to BMSC-Exos combined with $\beta$-TCP scaffolds in 
repairing critical-sized bone defects by promoting new bone regeneration and neovascularization in the defect area.

\section{DATA AVAILABILITY STATEMENT}

All datasets presented in this study are included in the article/supplementary material.

\section{ETHICS STATEMENT}

The animal study was reviewed and approved by The Ethics Committee of the Shanghai General Hospital, Shanghai Jiao Tong University School of Medicine.

\section{REFERENCES}

Behrend, C., Carmouche, J., Millhouse, P. W., Ritter, L., Moskal, J., Rubery, P., et al. (2016). Allogeneic and autogenous bone grafts are affected by historical donor environmental exposure. Clin. Orthop. Relat. Res. 474, 1405-1409. doi: 10.1007/s11999-015-4572-7

Benic, G. I., and Hämmerle, C. H. F. (2014). Horizontal bone augmentation by means of guided bone regeneration. Periodontol 2000 66, 13-40. doi: 10.1111/ prd.12039

Bianco, A. M., Uno, M., Oba-Shinjo, S. M., Clara, C. A., de Almeida Galatro, T. F., Rosemberg, S., et al. (2015). CXCR7 and CXCR4 expressions in infiltrative astrocytomas and their interactions with HIF1 $\alpha$ expression and IDH1 mutation. Pathol. Oncol. Res. 21, 229-240. doi: 10.1007/s12253-014-9 813-7

Cai, T., Sun, D., Duan, Y., Wen, P., Dai, C., Yang, J., et al. (2016). WNT/ $\beta$-catenin signaling promotes VSMCs to osteogenic transdifferentiation and calcification through directly modulating Runx2 gene expression. Exp. Cell. Res. 345, $206-$ 217. doi: 10.1016/j.yexcr.2016.06.007

Costa, V., Raimondi, L., Conigliaro, A., Salamanna, F., Carina, V., De Luca, A., et al. (2017). Hypoxia-inducible factor $1 \mathrm{~A}$ may regulate the commitment of mesenchymal stromal cells toward angio-osteogenesis by mirna-675-5P. Cytotherapy 19, 1412-1425. doi: 10.1016/j.jcyt.2017.09.007

Ding, H., Gao, Y.-S., Hu, C., Wang, Y., Wang, C.-G., Yin, J.-M., et al. (2013). HIF-1 $\alpha$ transgenic bone marrow cells can promote tissue repair in cases of corticosteroid-induced osteonecrosis of the femoral head in rabbits. PLOS ONE 8:e63628. doi: 10.1371/journal.pone.0063628

Fu, H., Deng, C., Teng, L., Cai, Z., Chen, J., and Lu, G. (2018). Effect of heparan sulfate mimetics from Escherichia coli K5 polysaccharide on SDF-1/CXCL12induced endothelial progenitor cells in vitro. Int. J. Biol. Macromol. 107, 2492-2500. doi: 10.1016/j.ijbiomac.2017.10.132

Gao, C., Deng, Y., Feng, P., Mao, Z., Li, P., Yang, B., et al. (2014). Current progress in bioactive ceramic scaffolds for bone repair and regeneration. Int. J. Mol. Sci. 15, 4714-4732. doi: 10.3390/ijms15034714

Jin, H., Liu, M.-L., Kim, H. A., Lee, M., An, S., Oh, J., et al. (2009). Role of the oxygen-dependent degradation domain in a hypoxia-inducible gene expression system in vascular endothelial growth factor gene therapy. Spine 34, E952E958.

Ju, C., Shen, Y., Ma, G., Liu, Y., Cai, J., Kim, I.-M., et al. (2018). Transplantation of cardiac mesenchymal stem cell-derived exosomes promotes repair in ischemic myocardium. J. Cardiovasc. Transl. Res. 11, 420-428. doi: 10.1007/s12265-0189822-0

Kawakami, Y., Ii, M., Matsumoto, T., Kuroda, R., Kuroda, T., Kwon, S.-M., et al. (2015). SDF-1/CXCR4 axis in Tie2-lineage cells including endothelial progenitor cells contributes to bone fracture healing. J. Bone. Miner. Res. 30, 95-105. doi: $10.1002 / j b m r .2318$

\section{AUTHOR CONTRIBUTIONS}

$\mathrm{XQ}$ and DH designed the tests. RW and ZW carried out the tests. CY analyzed experimental results. JT directed the experiments. CY and RW wrote this manuscript, two authors contributed equally to this work. Authors mentioned before modified this manuscript. WY, JZ and CQY provided valuable comments on the revised manuscript after submission.

\section{FUNDING}

This study was supported by the Cross Translational Medicine Research Fund of Shanghai Jiao Tong University (ZH2018QNA19), and the National Nature Science Foundation of China (Grant Nos. 81702144, 81702137, and 81972055).

Kim, D.-H., Lee, S.-H., Chi, S.-W., Nam, K. H., and Han, K.-H. (2009). Backbone resonance assignment of a proteolysis-resistant fragment in the oxygendependent degradation domain of the hypoxia inducible factor lalpha. Mol. Cells 27, 493-496. doi: 10.1007/s10059-009-0065-4

Kwon, T.-G., Zhao, X., Yang, Q., Li, Y., Ge, C., Zhao, G., et al. (2011). Physical and functional interactions between Runx 2 and HIF- $1 \alpha$ induce vascular endothelial growth factor gene expression. J. Cell Biochem. 112, 3582-3593. doi: 10.1002/ jcb.23289

Lee, S.-H., Che, X., Jeong, J.-H., Choi, J.-Y., Lee, Y.-J., Lee, Y.-H., et al. (2012). Runx2 protein stabilizes hypoxia-inducible factor- $1 \alpha$ through competition with von hippel-lindau protein (pVHL) and stimulates angiogenesis in growth plate hypertrophic chondrocytes. J. Biol. Chem. 287, 14760-14771. doi: 10.1074/jbc. m112.340232

Lee, Y.-H., Park, H.-K., Auh, Q. S., Nah, H., Lee, J. S., Moon, H.-J., et al. (2020). Emerging potential of exosomes in regenerative medicine for temporomandibular joint osteoarthritis. Int. J. Mol. Sci. 21:1541. doi: 10.3390/ ijms21041541

Li, D., Zhang, P., Yao, X., Li, H., Shen, H., Li, X., et al. (2018). Exosomes derived from miR-133b-modified mesenchymal stem cells promote recovery after spinal cord injury. Front Neurosci 12:845. doi: 10.3389/fnins.2018.00845

Lin, L., Shen, Q., Leng, H., Duan, X., Fu, X., and Yu, C. (2011). Synergistic inhibition of endochondral bone formation by silencing Hifl $\alpha$ and Runx2 in trauma-induced heterotopic ossification. Mol. Ther. 19, 1426-1432. doi: 10. 1038/mt.2011.101

McGovern, J. A., Griffin, M., and Hutmacher, D. W. (2018). Animal models for bone tissue engineering and modelling disease. Dis. Model Mech. 11:33084.

Nakamura, Y., Miyaki, S., Ishitobi, H., Matsuyama, S., Nakasa, T., Kamei, N., et al. (2015). Mesenchymal-stem-cell-derived exosomes accelerate skeletal muscle regeneration. FEBS Lett. 589, 1257-1265. doi: 10.1016/j.febslet.2015.03.031

Oryan, A., and Alidadi, S. (2018). Reconstruction of radial bone defect in rat by calcium silicate biomaterials. Life Sci. 201, 45-53. doi: 10.1016/j.lfs.2018. 03.048

Phinney, D. G., and Pittenger, M. F. (2017). Concise review: MSC-derived exosomes for cell-free therapy. Stem Cells 35, 851-858. doi: 10.1002/stem. 2575

Phinney, D. G., and Prockop, D. J. (2007). Concise review: mesenchymal stem/multipotent stromal cells: the state of transdifferentiation and modes of tissue repair-current views. Stem Cells 25, 2896-2902. doi: 10.1634/stemcells. 2007-0637

Poe, A. J., and Knowlton, A. A. (2018). Exosomes and cardiovascular cell-cell communication. Essays Biochem. 62, 193-204. doi: 10.1042/ebc20170081

Qi, X., Danping, L., Zhao, H., and Zhang, J. (2013). ExPerimental study of transplantation of rabbit endothelial progenitor cells transfected by Ad-BMP-2IRES-HIF- $1 \alpha \mathrm{mu}$ for treatment of avascularnecrosis of the femoral head. Chinese J. Cell Bio. 35, 967-972. 
Reinisch, A., Etchart, N., Thomas, D., Hofmann, N. A., Fruehwirth, M., Sinha, S., et al. (2015). Epigenetic and in vivo comparison of diverse MSC sources reveals an endochondral signature for human hematopoietic niche formation. Blood 125, 249-260. doi: 10.1182/blood-2014-04-572255

Roccaro, A. M., Sacco, A., Maiso, P., Azab, A. K., Tai, Y.-T., Reagan, M., et al. (2013). BM mesenchymal stromal cell-derived exosomes facilitate multiple myeloma progression. J. Clin. Invest. 123, 1542-1555. doi: 10.1172/jci66517

Rogers, G. F., and Greene, A. K. (2012). Autogenous bone graft: basic science and clinical implications. J. Craniofac. Surg. 23, 323-327. doi: 10.1097/scs. 0b013e318241dcba

Satija, N. K., Singh, V. K., Verma, Y. K., Gupta, P., Sharma, S., Afrin, F., et al. (2009). Mesenchymal stem cell-based therapy: a new paradigm in regenerative medicine. J. Cell Mol. Med. 13, 4385-4402.

Takeuchi, R., Katagiri, W., Endo, S., and Kobayashi, T. (2019). Exosomes from conditioned media of bone marrow-derived mesenchymal stem cells promote bone regeneration by enhancing angiogenesis. PLOS ONE 14:e0225472. doi: 10.1371/journal.pone.0225472

Tan, S. H. S., Tjio, C. K. E., Wong, J. R. Y., Wong, K. L., Chew, J. R. J., Hui, J. H. P., et al. (2020). Mesenchymal stem cell exosomes for cartilage regeneration: a systematic review of preclinical in vivo studies. Tissue Eng. Part. B Rev. 2020:326.

Thiagarajan, L., Abu-Awwad, H. A.-D. M., and Dixon, J. E. (2017). Osteogenic programming of human mesenchymal stem cells with highly efficient intracellular delivery of RUNX2. Stem Cells Transl. Med. 6, 2146-2159. doi: 10.1002/sctm.17-0137

Vega, O. A., Lucero, C. M. J., Araya, H. F., Jerez, S., Tapia, J. C., Antonelli, M., et al. (2017). Wnt/ $\beta$-Catenin signaling activates expression of the bone-related transcription factor RUNX2 in select human osteosarcoma cell types. J. Cell Biochem. 118, 3662-3674. doi: 10.1002/jcb.26011

Wang, J., Bonacquisti, E. E., Brown, A. D., and Nguyen, J. (2020). Boosting the biogenesis and secretion of mesenchymal stem cell-derived exosomes. Cells 9:660. doi: 10.3390/cells9030660

Wang, Y., Han, Z.-B., Song, Y.-P., and Han, Z. C. (2012). Safety of mesenchymal stem cells for clinical application. Stem Cells Int. 2012:652034.

Wei, C.-C., Lin, A. B., and Hung, S.-C. (2014). Mesenchymal stem cells in regenerative medicine for musculoskeletal diseases: bench, bedside, and industry. Cell Transplant. 23, 505-512. doi: 10.3727/096368914x678328

Wei, L., Lu, J., Feng, L., Li, S., Shan, J., and Li, Y. (2010). Construction of recombinant adenovirus vector containing a modified gene that codes for human hypoxia-inducible factor-1alpha without oxygen-dependent degradation domain. Plasmid 63, 20-26. doi: 10.1016/j.plasmid.2009.09.002

Xu, T., Luo, Y., Wang, J., Zhang, N., Gu, C., Li, L., et al. (2020). Exosomal miRNA128-3p from mesenchymal stem cells of aged rats regulates osteogenesis and bone fracture healing by targeting Smad5. J. Nanobiotechnology 18:47.

Xue, C., Shen, Y., Li, X., Li, B., Zhao, S., Gu, J., et al. (2018). Exosomes derived from hypoxia-treated human adipose mesenchymal stem cells enhance angiogenesis through the PKA Signaling pathway. Stem Cells Dev. 27, 456-465. doi: 10.1089/ scd.2017.0296
Yang, C., Liu, H., and Liu, D. (2014). Mutant hypoxia-inducible factor $1 \alpha$ modified bone marrow mesenchymal stem cells ameliorate cerebral ischemia. Int. J. Mol. Med. 34, 1622-1628. doi: 10.3892/ijmm.2014.1953

Yellowley, C. E., and Genetos, D. C. (2019). Hypoxia Signaling in the skeleton: implications for bone health. Curr. Osteoporos. Rep. 17, 26-35. doi: 10.1007/ s11914-019-00500-6

Zhang, B., Tian, X., Hao, J., Xu, G., and Zhang, W. (2020). Mesenchymal stem cell-derived extracellular vesicles in tissue regeneration. Cell Transplant. 29:963689720908500.

Zhang, J., Feng, Z., Wei, J., Yu, Y., Luo, J., Zhou, J., et al. (2018). Repair of critical-sized mandible defects in aged rat using hypoxia preconditioned BMSCs with Up-regulation of Hif-1 $\alpha$. Int. J. Biol. Sci. 14, 449-460. doi: 10.7150/ijbs. 24158

Zhang, L., Jiao, G., Ren, S., Zhang, X., Li, C., Wu, W., et al. (2020). Exosomes from bone marrow mesenchymal stem cells enhance fracture healing through the promotion of osteogenesis and angiogenesis in a rat model of nonunion. Stem Cell Res. Ther. 11:38.

Zhang, S., Chuah, S. J., Lai, R. C., Hui, J. H. P., Lim, S. K., and Toh, W. S. (2018). MSC exosomes mediate cartilage repair by enhancing proliferation, attenuating apoptosis and modulating immune reactivity. Biomaterials 156, 16-27. doi: 10.1016/j.biomaterials.2017.11.028

Zhang, W., Feng, C., Yang, G., Li, G., Ding, X., Wang, S., et al. (2017). 3D-printed scaffolds with synergistic effect of hollow-pipe structure and bioactive ions for vascularized bone regeneration. Biomaterials 135, 85-95. doi: 10.1016/j. biomaterials.2017.05.005

Zhao, G., Ge, Y., Zhang, C., Zhang, L., Xu, J., Qi, L., et al. (2020). Progress of mesenchymal stem cell-derived exosomes in tissue repair. Curr. Pharm. Des. 26, 2022-2037. doi: 10.2174/1381612826666200420144805

Zhao, S., Zhang, J., Zhu, M., Zhang, Y., Liu, Z., Tao, C., et al. (2015). Threedimensional printed strontium-containing mesoporous bioactive glass scaffolds for repairing rat critical-sized calvarial defects. Acta. Biomater 12, 270-280. doi: 10.1016/j.actbio.2014.10.015

Zhao, Z., Ma, X., Ma, J., Sun, X., Li, F., and Lv, J. (2018). Naringin enhances endothelial progenitor cell (EPC) proliferation and tube formation capacity through the CXCL12/CXCR4/PI3K/Akt signaling pathway. Chem. Biol. Interact. 286, 45-51. doi: 10.1016/j.cbi.2018.03.002

Conflict of Interest: The authors declare that the research was conducted in the absence of any commercial or financial relationships that could be construed as a potential conflict of interest.

Copyright (c) 2020 Ying, Wang, Wang, Tao, Yin, Zhang, Yi, Qi and Han. This is an open-access article distributed under the terms of the Creative Commons Attribution License (CC BY). The use, distribution or reproduction in other forums is permitted, provided the original author(s) and the copyright owner(s) are credited and that the original publication in this journal is cited, in accordance with accepted academic practice. No use, distribution or reproduction is permitted which does not comply with these terms. 